\title{
The role of the public in developing interventions: a reflection and critique of a cancer clinical trials unit's model
}

This article was published in the following Dove Press journal:

Patient Preference and Adherence

4 November 2014

Number of times this article has been viewed

\author{
Jim Fitzgibbon' \\ Jessica Baillie' \\ Natalie Simon ${ }^{2}$ \\ Annmarie Nelson' \\ 'Marie Curie Palliative Care Research \\ Centre, School of Medicine, Cardiff \\ University, Cardiff, Wales; ${ }^{2}$ Involving \\ People, National Institute for Social \\ Care and Health Research Clinical \\ Research Centre, Cardiff, Wales
}

Correspondence: Annmarie Nelson Marie Curie Palliative Care Research Centre, School of Medicine, Cardiff University, First Floor Neuadd Meirionnydd, Heath Park, Cardiff CFI 4 4YS, Wales

Tel +442920687473

Email nelsonA9@cf.ac.uk

\begin{abstract}
The importance of involving lay representatives in research is well-recognized but is not consistently meaningfully practiced or reported. Although the positive outcomes of lay representative involvement can include more relevant research questions and outcomes, challenges are also apparent, including tokenistic involvement by research teams. A Cancer Research UK-funded and National Cancer Research Institute-registered cancer clinical trials unit in the United Kingdom established a program of work to promote genuine and consistent involvement of lay representatives (or "research partners") as part of the research team. Furthermore, a volunteer was employed to recruit and coordinate the research partners in partnership with a national agency for public involvement in health and social care research in Wales. This article reports on the development of this project and how it will be formally evaluated. Recommendations for involving lay representatives are also posed.
\end{abstract}

Keywords: public involvement, lay representative, clinical trials unit

\section{Introduction}

The aim of this article is to place within a national context an exploration and critique of one clinical trials unit's (CTU's) model for involving lay representatives in research, including cancer clinical trials with embedded qualitative studies. The Cancer Research UK-funded CTU is a National Cancer Research Institute (NCRI)accredited and UK Clinical Research Collaboration (CRC)-registered unit. The CTU was established in 2005 and was joined by a palliative care research center in 2010, with a United Kingdom-wide remit to develop the palliative care clinical trials portfolio and to undertake methodological and academic research. The unit has a clear framework and program for involving lay representatives in all appropriate research studies, including determining which studies to adopt and developing, running, reporting, and overseeing studies. The involvement of lay representatives within the unit is coordinated by a senior member staff and a volunteer research partner coordinator based with the unit part-time.

This article presents an exemplar of best practice, exploring and critiquing the CTU's model for involving lay representatives in all aspects of research within the unit, and it also includes a personal reflection on the role of the research partner coordinator, developed in partnership with a national agency for public involvement in health and social care research in Wales. To place this initiative into context, the article will first define lay representative involvement before providing an overview of how the concept emerged and is now practiced. 


\section{Overview of lay representative involvement \\ Defining involvement}

Public, service user, or lay representative involvement in research can hold different meanings for different people. For clarity, we use the term lay representative throughout the first section of this article to describe a person who is involved as a member of a research team, whether or not they have accessed health or social care.

INVOLVE $^{1}$ and Involving People, ${ }^{2}$ which are organizations in England and Wales, respectively, that encourage public involvement in research, specify it involves research being carried out "with" or "by" the public. Morrow et al define "service user involvement in research" as a situation in which service users and researchers work together to design, undertake, or evaluate research, rather than service users simply being subjects of the research. ${ }^{3}$ This definition is reflected in the Research Governance Frameworks for England ${ }^{4}$ and Wales, ${ }^{5}$ which advocate the involvement of lay representatives in research.

\section{Development of lay representative involvement}

The practice of involving lay representatives in research appeared to develop in the wake of increasing recognition that patients should be more actively involved in decisions about their healthcare. An early example includes the Community Health Councils, established in 1974 to represent patients' perspectives in healthcare provision. ${ }^{6}$ Public involvement was well established within health and social care policy by the time of the National Health Service Act 2006 in England. Furthermore, recent Department of Health initiatives focus on patients sharing decision-making with clinicians, advocating "no decision about me without me." " Torrance and Wilson" discusses embracing the voice of the public, ensuring shared responsibility for decisions surrounding health and social care provision and how this is critical during recession, when services may be curtailed because of financial limitations.

Morrow et $\mathrm{al}^{3}$ provide a useful overview of the development of lay representative involvement in research, tracking its evolution with historical and cultural developments. They suggest that the human rights movement in the United States in the 1960s, and public concern for the running of health services in the 1970s, led to the first public involvement in research in the 1980s. ${ }^{3}$ The 1980s also witnessed high-profile exposure of research scandals, demonstrating the lack of research governance. ${ }^{3}$ Beresford ${ }^{10}$ explores the emergence of lay representative involvement, describing the need in the 1980s for services to become more "user-centered and user-led," rather than prescribed by the service provider. User involvement became the uniting idea supporting this development. ${ }^{11}$ Finally, a change of terminology in the 1980s and 1990s led to research "subjects" being referred to as "participants," suggesting they had a more active role in the process.

There is a large and growing body of literature considering community-based participatory health research. Participatory health research is seen as a key methodological approach to public involvement. ${ }^{12}$ The goal of participatory research is to negotiate a balance between "developing valid generalizable knowledge and benefiting the community being researched." ${ }^{13} \mathrm{~A}$ common aspect of participatory research is that the community or the affected population should play a leading role in the research process. ${ }^{14}$ In 2009, the International Collaboration on Participatory Health Research was established to promote the use of participatory health research in health-related decision-making and research design. ${ }^{15}$

With the increasing impetus to include lay representatives in research as members of the research team, the NCRI, which is responsible for planning and coordinating cancer research in the United Kingdom, demonstrate their commitment to public involvement through the inclusion of at least two lay representatives on each clinical studies group. Schemes in the United Kingdom provide guidance and assistance on public involvement in health and social care research, including Involving People (Wales) and INVOLVE (England). However, at present, Northern Ireland and Scotland do not have equivalent organizations to support the involvement of lay representatives in research.

INVOLVE ${ }^{16}$ suggests a cycle for involving members of the public in research, which includes them as partners throughout the research process, from identifying and prioritizing to commissioning, designing and managing, undertaking, disseminating, implementing, and evaluating research. In reality, members of the public, patients, and carers have been involved in a wide variety of research projects as participants, ${ }^{17}$ members of the research team, ${ }^{18,19}$ and researchers. ${ }^{20}$ Morrow et $\mathrm{al}^{21}$ describe different theoretical underpinnings of public involvement, from "emancipatory movements" to "top down drives." ${ }^{21}$ Different theoretical perspectives have led to a variety of involvement activities, ranging from discrete public consultations to sustained partnerships involving people with experience of specific health conditions. ${ }^{22}$

To constitute a patient-centered research, Tischler et a ${ }^{17}$ argue that one of three elements should be included: to 
address the lived experience of patients, to consider patients' expectations of illness/treatment, or to consider patients' opinions of the research process. However, their mixedmethods study with psychiatrists and mental health service users in the United Kingdom identified that for research to be patient-centered, participants felt they should be involved at all stages of the research as collaborators. ${ }^{17}$ Wilson et al ${ }^{20}$ adopted an emancipatory approach in which they established a group in Wales providing education and empowerment training to mental health service users about the research process. Participants evaluated the training positively, and Wilson et $\mathrm{al}^{20}$ asserted they had provided the basis for service users to engage with research and researchers. Smith et al ${ }^{19}$ used a different approach and recruited representatives from 24 organizations in England that advocate for service users to guide the development and undertaking of a systematic review. Finally, Barber et $\mathrm{al}^{18}$ included service users as part of an advisory group that guided a Delphi consensus process in the United Kingdom, later qualitatively exploring their perspectives of being involved in the research. There are thus varying approaches to including lay representatives in research, and different approaches, or levels of involvement, will be appropriate for different research questions and methods.

\section{Outcomes of lay representative involvement}

Morrow et $\mathrm{l}^{3}$ highlight that involving lay representatives in research should not be an "add-on," and in fact, the relationship is complex and challenging. Researchers hold different views about the value of involving lay representatives, but less is known about service users' views and expectations of being involved in research. ${ }^{3}$

\section{Research}

There is growing interest considering the effect of lay representative involvement on research. Benefits include ensuring that research questions ${ }^{3}$ and outcomes are patient-relevant, ${ }^{17}$ with service users in Wilson et al' $\mathrm{s}^{20}$ and Cotterell et al' $\mathrm{s}^{23}$ projects arguing that they are aware of how services need to be improved. Boote et al's ${ }^{12}$ lay representative involvement in health research review identified questions in need of attention, including the influence of public involvement on health research. Brett et al's ${ }^{24}$ recent systematic review of the international literature explored the effect on research of involving lay representatives, including benefits and challenges. Positive effects of lay representative involvement were apparent throughout the research process, including, for example, identifying relevant research topics, ensuring language is understandable, identifying appropriate ways of accessing participants, increasing credibility of results during data analysis, and influencing the implementation of research findings. ${ }^{24}$ However, Brett et $\mathrm{al}^{24}$ also identified challenges in involving lay representatives in research, including perfunctory involvement of representatives, low attendance rates at meetings, and time and cost burdens to studies.

Lay representative involvement is central to health and social care research policies, and research commissioners are supporting it financially, although the evidence evaluating its effect remains weak. ${ }^{24}$

\section{Lay representatives}

The benefits of involving lay representatives in research appear to be varied. In one study in a mental health setting, service users reported feeling valued and respected, as well as positive about assuming a role in the research team, and psychiatrists also evaluated the experience positively. ${ }^{17}$ Lay representatives in Cotterell et al' $\mathrm{s}^{23}$ study reported that being involved as researchers had improved their experiences of living with cancer, reducing their feelings of hopelessness. Furthermore, lay representatives reported benefits of their involvement in research, which included seeing the effect of the research, sharing their experience, and increasing knowledge and confidence, whereas researchers reported positively the diverse perspectives that patients brought to the research. ${ }^{25}$ There is thus some agreement that involving lay representative in research is beneficial for the public, the researchers, and the research itself.

However, one challenge for involving people in research is the uncertainty about how to best involve groups of service users in research projects for different purposes. ${ }^{3}$ Individuals who were involved in research in Cotterell et al' ${ }^{23}$ study were concerned that their involvement was only minimal, they felt uncertain about what was expected of them, and they were distressed at times about how cancer was discussed by healthcare professionals. In addition, they felt marginalized and undervalued by staff. ${ }^{23}$ Involving People evaluated their program to assess whether they had met their aim of supporting public involvement in research, highlighting challenges faced by patients involved in research in Wales. ${ }^{25}$ Their evaluation revealed perceived barriers that prevented the individuals' participation in research, including struggling to find carers or transport, limited opportunities for participation, and use of jargon by researchers. ${ }^{25}$ Therefore, this report further highlights the importance of understanding 
the barriers that could prevent patients from being successfully included.

This section has provided a brief overview of the variety and scope of lay representative involvement in research. It is clear that there is a motivation by members of the public to participate in health research as equal members of the team, rather than purely as participants, although there can be both professional and organizational barriers that prohibit their involvement. The next section focuses on how a CTU developed a program for involving lay representatives as members of the research team.

\section{Development of lay representative involvement in a CTU: research partners \\ History of lay representative involvement in the CTU}

The CTU discussed here, funded by Cancer Research UK, was established in 2005, and from its genesis, the importance of involving lay representatives in research was recognized. Cancer Research UK cites the importance of engaging the public in their research strategy. ${ }^{26}$ Between 2005 and 2012, the CTU, in association with other organizations, strategically developed how lay representatives were involved in research. This is depicted in Figure 1.

By 2008, in line with the example set by the NCRI, ${ }^{27}$ lay representatives were included as members of the Trial Management Groups (TMG), reviewing documents, contributing to meetings, and chairing and presenting at subcommittees. However, these individuals were often previous patients and were recruited opportunistically by the trial's chief investigator; there was no formal process for recruiting, training, or monitoring, although travel reimbursement and an honorarium were available to representatives, funded through trial budgets. From a CTU perspective, there was concern that future service users could potentially be recruited merely as part of a tick-box process. Ethical approvals and grant applications require that studies have lay representative input, and the race to deadlines had the potential for their involvement to be included as an afterthought. In the context of a trials unit, it is essential that lay representatives are recruited to perform specific tasks and are equipped to operate with confidence and equality in a highly rigorous and scientific environment as valued members of interprofessional teams.

An increasing number of complex trials being coordinated by the CTU meant that a more professionally recruited and supported group of lay representatives would be needed as members of the research team. The CTU therefore convened a discussion group with organizations experienced at working with lay representatives and research networks, including the NCRI and Involving People (with CRC Cymru, which is now the National Institute for Social Care and Health Research CRC [NISCHR CRC]). It was agreed that a collaborative approach to involvement would be undertaken, including having Involving People providing funding and support toward the recruitment and administration of lay representatives, the NCRI offering advice about job descriptions for user representatives, and the CTU hosting and auditing the initiative.

\section{Initial audit and introducing research partners}

Of the eight funded clinical trials coordinated by the CTU in March 2009, all but one had patient representation (the single trial lacking representation did not need it because of nature of the trial). Several large trials (with more than 1,400 participants) had just one lay representative. Individual, informal interviews were undertaken by a senior staff member with several lay representatives in 2009 to discuss their roles, which highlighted the experience, trials knowledge, and motivation of the cohort. It was concluded that lay representatives were well experienced and did not require further training, but the CTU was aware that as the unit continued to expand, it was important to ensure that their involvement was meaningful and of high quality.

However, Involving People advised that two lay representatives, renamed research partners to represent their equal status within the research team, be included in each trial. The CTU worked closely with Involving People to ensure equitable recruitment of research partners. The Involving People network is a diverse network of members of the public (patients, carers, and service users), representing a range of different conditions (including cancer), diseases, skills, ages, disabilities, sexes, and races. The network is informed of a large number of public involvement opportunities in research, enabling individuals to have experience of public involvement and knowledge of research. Individuals are supported by Involving People through a training program and by covering expenses and offering payment for time and involvement. Research partners were recruited through advertisements posted through Involving People seeking applications from people with, principally, an interest in the particular field of research derived either from personal or familial experience. Other desirable attributes included the confidence or ability to present a layperson's view in 


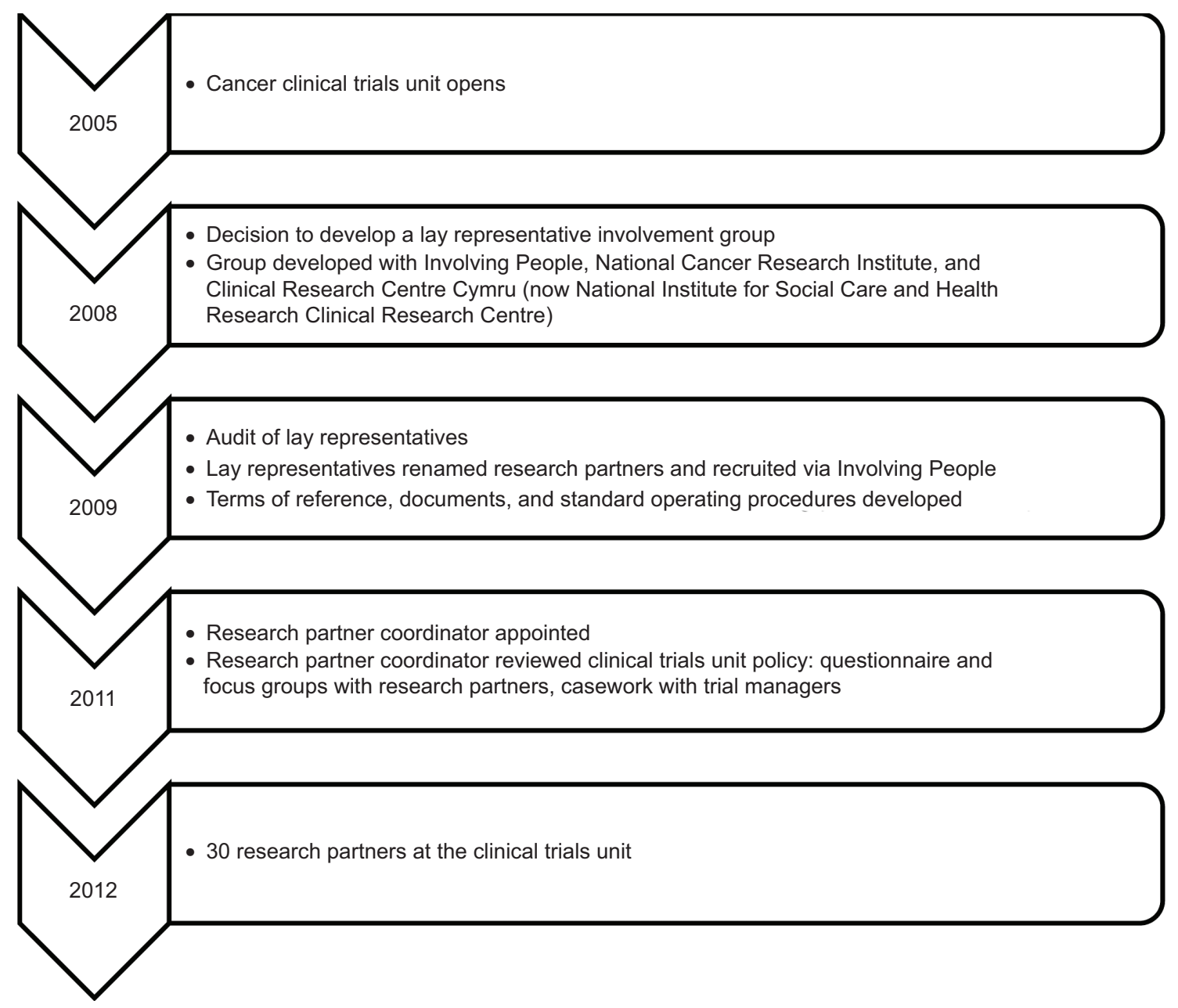

Figure I History of lay representative involvement at the clinical trials unit.

a professional environment and background knowledge of committee work and research. However, it was, above all, important that research partners had a willingness to learn and undertake appropriate training in areas in which they lacked confidence or knowledge.

Applicant research partners were interviewed by the appropriate trial manager and CTU senior staff member. Successful applicants were provided with information about the trial and what would be expected of them, a terms-ofreference document, an invitation to attend a consumer training workshop facilitated by Macmillan Cancer Support and the National Cancer Research Network, an information pack about cancer (from the United Kingdom Association of Cancer Registries Cancer Registration Development Group), and further training as requested.

New research partners were supported at their first TMG meeting by a separate trial manager, were encouraged to ask questions and contribute as equal members of the group, and afterward met with the CTU senior staff member to debrief.
Research partners' attendances at TMG meetings were documented in the Trial Master Files, and their expenses were reimbursed. They were asked to complete a feedback questionnaire, which asked research partners to comment on and describe their experience at TMG meetings, including whether they felt able to contribute to group discussions as an equal member of the group. To encourage a sustainable group of research partners and enable staff in the CTU to manage their role in this process, three standard operating procedures (SOPs) were developed to guide recruitment, budget costing and financing, and training and mentoring. This initiative was presented at national conferences. ${ }^{28,29}$

\section{Development of research partner involvement}

As the number of research partners increased and their contribution to the research process developed, the CTU and Involving People agreed that a volunteer research partner coordinator would be appointed to lead and advocate for the 
volunteers and research staff. In January 2011, a research partner coordinator, an experienced health research volunteer, was appointed via Involving People to work 2 days per month within the CTU, on a voluntary basis, to recruit and support research partners and oversee policy and practice in this area. This voluntary role was also, in part, a solution to the limited funding available for public involvement coordination across the research infrastructure in Wales. This post, a partnership between the CTU and Involving People, appears at the time of writing to be unique in the United Kingdom. Its uniqueness lies not in the nature of the post (there are at least two other CTUs that have made similar appointments) but in its decision to recruit a volunteer to fill it. The post-holder and senior staff member were invited to speak in quick turn at a number of conferences to share their experiences with other $\mathrm{CTUs}^{30}$ and to contribute to written advice for professionals. ${ }^{31}$

However, the potential challenges faced by the research partner coordinator included maintaining the trust of management as an organizational "cuckoo in the nest," neither formal employee nor traditional volunteer. He was also empowered to recruit new research partners to the organization with Involving People and to brief them as part of the induction process. In addition, the coordinator needed to gain acceptance among other professionals who may have had doubts about the ability of the post-holder to succeed in the role. Crucially, he had to empathize with professionals' as well as lay representatives' perspectives, and for this reason, the early title of the post (research partner champion) was set aside. Finally, the research partner coordinator was required to establish administrative support structures in a clinical trial environment that had not previously had to provide office space or security access for a volunteer.

One priority of the research partner coordinator was to review the CTU's policy and practice in relation to research partners. This review involved seeking research partners' experiences through a questionnaire and a small focus group. Individual pieces of casework, as exemplars of practice, with trial managers and research partners also informed the review. Recommendations arising from the review included clarifying what was expected of research partners documented in CTU SOPs; reaffirming the prime responsibility of trial managers for gathering the personal data of research partners working on their trial and supporting them; improving communication with research partners via trial newsletters and a semi-annual research partner newsletter; simplifying the payment system for research partners; inviting research partners to the CTU's annual celebration; and encouraging research partners to undertake training with Involving People and, in particular, good clinical practice (research training) that all researchers attend.

All but one of these recommendations (annual celebration) were implemented within 12 months. In November 2012, the CTU had recruited 30 research partners in partnership with Involving People to ensure sufficient research partner representation across all research studies.

\section{How research partners are involved in research}

In line with the research cycle advocated by INVOLVE, ${ }^{16}$ research partners are involved throughout the research process by the CTU and are recruited when the trial is being set up, often before the study has been funded. Figure 2 depicts the process of research partner recruitment and involvement. The implementation of three SOPs relating to research partner involvement has become routine within the organization and is subject to regular annual review in partnership with Involving People. Research partners are supported by staff at the highest level, as evidenced by the research partner coordinator's membership on the unit's trial review group and the coordinator's involvement in applying for ongoing research funding for the unit.

Trial managers endeavor to engage research partners at the earliest possible stage of trial development, including as coapplicants on grants, and through the new induction process, to support them throughout their work. Research partners participate in TMG and trial steering committee meetings, review study documentation before seeking ethical approval, and contribute to annual progress reviews of all studies (where decisions about the efficacy of continuing with a trial is determined). Finally, research partners are included when disseminating study results via conferences and journals, as appropriate. ${ }^{32,33}$

\section{Looking forward: the future of research partner involvement at the CTU}

The CTU's work to engage lay representatives in its research has been developed extensively. Nevertheless, the CTU acknowledges that there remains much to do to achieve full and meaningful engagement of the public in its work. Reference has been made earlier to the 2011 review of its policy and practice. Although most of its recommendations were implemented, one remains to be so: an annual celebration of research partners' work. In addition, the most recent joint review with Involving People (December 2012) identified further issues that need to be addressed, including revised induction processes from the research partner coordinator and 


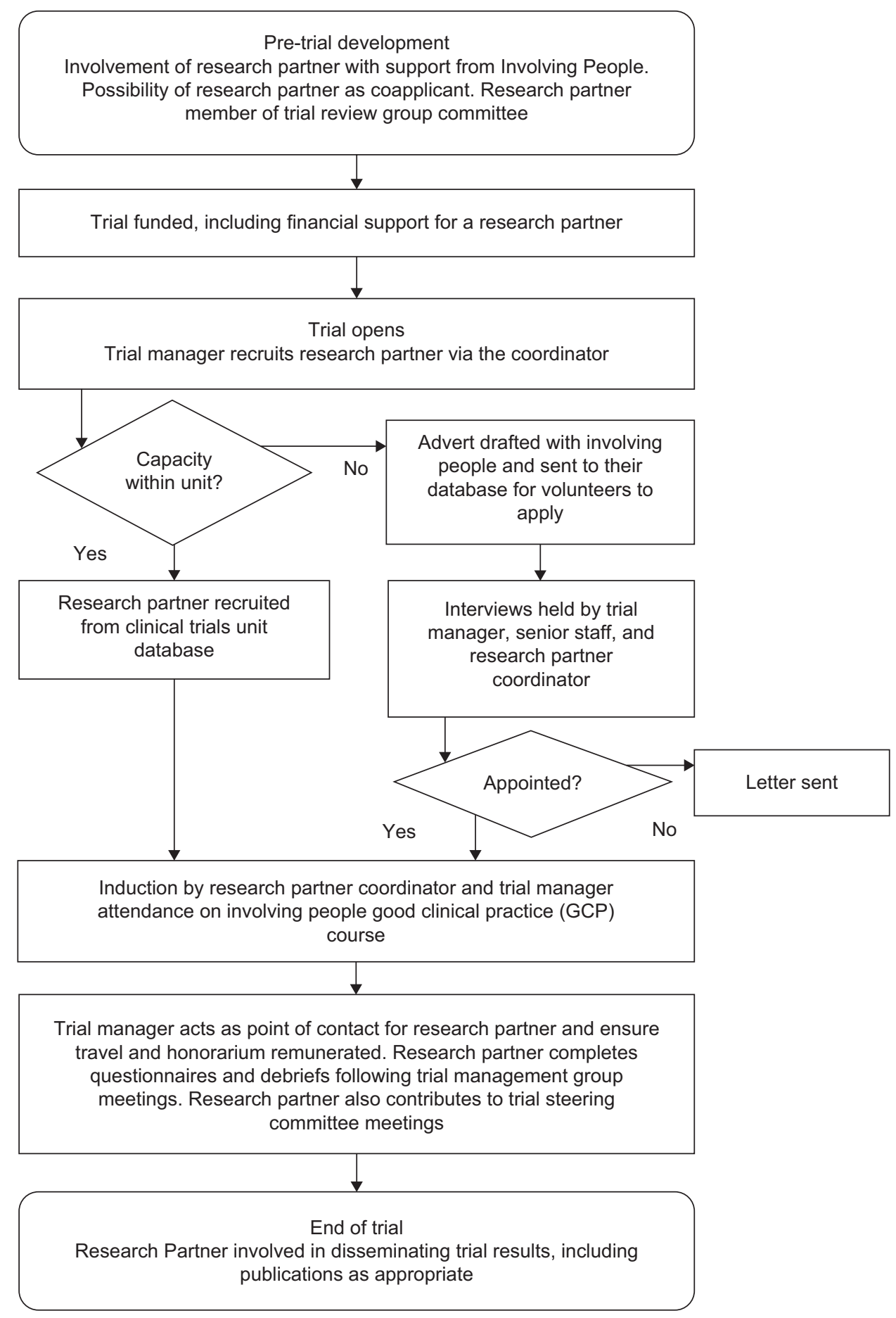

Figure 2 Recruitment and involvement of research partners at the clinical trials unit.

trial manager, ensuring that research partners receive support and training from the CTU and Involving People and ensuring the seeking of contributions to the unit's newsletter from all research partners. The agreed timescale for these initiatives was 12 months, in advance of the next review.

The research partner coordinator's presence in the CTU has also prompted other, broader initiatives to be implemented. CTU volunteers are rightly regarded as fellow professionals within the unit, and its recruitment, induction of, and support for them seeks to reflect this. Research partners are encouraged via a questionnaire to provide feedback on their experience of the CTU. However, there is, to date, no formal system to provide feedback on the quality of their contribution to the work. After discussion with Involving 
People, it was agreed that this was an issue best dealt with nationally with a contribution from the CTU. Furthermore, there needs to be further refinement of the CTU's already rigorous identification of patient-focused research priorities in partnership with lay representative agencies. The importance of including lay representatives when prioritizing research topics has been identified in the wider literature ${ }^{24,34}$ but is in its infancy at the CTU. This may well result in further refinement of the CTU's research partner involvement policy. In addition, the CTU hopes to quantify the effect of research partners on its research. There is growing interest in the effect of lay representative involvement in research, on which there has been limited robust research. ${ }^{24,35}$ Exploring the effect of lay representative involvement in research was the subject of a presentation by the CTU at Involving People's 2013 Annual General Meeting, and one of the research partners presented her experiences in the role at the NISCHR CRC Conference 2013. However, there is a desire to undertake more work on this locally, as a joint initiative between the CTU and Involving People.

The CTU and Involving People finally need to consider recruitment of the research partner coordinator's successor. Although being the coordinator is a demanding and challenging role, the Involving People network members are experienced health research volunteers, and it is thus anticipated that the successor would be recruited through this network.

\section{Conclusion}

Recognizing the value of public involvement in research, the CTU has sought to ensure the genuine engagement of lay representatives in its work. Its policy and practices over the course of 9 years are evidence of its commitment to lay representative involvement. The last 3 years, partly with the establishment of the post of volunteer research partner coordinator, have seen some very significant steps forward in this work. The CTU now has a process for regular review of the research partner program, including reviewing the research partner coordinator role with Involving People and a broadening of the lay representative involvement agenda with clear priorities for the future.

A recent meeting of senior members of the research community from across the United Kingdom has begun to look at all aspects of randomized controlled trial processes, with public and patient involvement as an identified component. ${ }^{34}$ The initiative will invite the research community to follow a five-step process to identify current practice and evidence gaps and to undertake evaluations of improvements made. ${ }^{34}$
Interest in meaningful PPI is increasing, and dissemination of exemplars of practice is much needed.

Opportunities for public involvement in research are growing, and there is a growing body of literature in this area; however, one may argue that the literature on the effect it makes is a shortcoming. Involving People and the CTU recognize the value of conducting a full evaluation of the effect of public involvement in its work. This might take the form of a realist evaluation; for example, to study the effect of the coordinator's role on the quality of public involvement across the unit. Results could add to the growing body of literature on public involvement in participatory health research. Barber et $\mathrm{al}^{35}$ considered the feasibility of such evaluation, concluding it may be possible to evaluate the effect of lay representative involvement on "research processes, outcomes and on key stakeholders." However, this is still an undeveloped area of research, and there is a pressing need to explore and develop structured approaches to evaluate and report on the investments being made.

As a result of this program of work, recommendations for meaningful involvement of lay representatives within clinical trials include ensuring a senior member of staff assumes responsibility for embedding and overseeing the involvement of lay representatives across a portfolio of studies and offers support to individuals undertaking the role; working jointly with national agencies to support recruitment, training, and development of lay representatives; appointing a volunteer who is able to be an advocate for lay representatives within a unit and act as a point of contact for staff within the unit and volunteers, ensuring they are adequately mentored and supported to fulfil this role; and promoting a culture of consistent involvement of lay representatives throughout the research process, through membership on trial committees and the development of SOPs.

\section{Disclosure}

The authors report no conflicts of interest in this work.

\section{References}

1. INVOLVE. INVOLVE Strategy 2012-2015: Putting people first in research. London: National Institute for Health Research; 2012. Available from: http://www.invo.org.uk/wp-content/uploads/2012/04/ INVOLVEStrategy2012-15.pdf. Accessed March 2014.

2. Involving People. About Us. Available from: http://www.wales.nhs.uk/ sites3/page.cfm?orgid=1023\&pid=59258. Accessed March 2014 .

3. Morrow E, Boaz A, Brearley S, et al. Handbook of Service User Involvement in Nursing and Healthcare Research. Chichester: Wiley-Blackwell; 2011.

4. Department of Health. Research governance framework for health and social care. 2nd ed. London: Department of Health; 2005. Available from: https://www.gov.uk/government/uploads/system/uploads/ attachment_data/file/139565/dh_4122427.pdf. Accessed July 2014. 
5. Welsh Assembly Government. Research Governance Framework for Health and Social Care in Wales. 2nd ed. Cardiff, United Kingdom: Wales Office of Research and Development for Health and Social Care. Available from: http://www.wales.nhs.uk/sites3/documents/952/ Research\%20Governance\%20Framework\%202009\%20(English)1.pdf. Accessed July 2014.

6. House of Commons Health Committee. Patient and Public Involvement in the NHS. London: The Stationary Office; 2007.

7. United Kingdom. National Health Service Act 2006. Available from: http://www.legislation.gov.uk/ukpga/2006/41/contents. Accessed July 2014.

8. Department of Health. Equity and Excellence: Liberating the NHS. London: Department of Health; 2010. Available from: https://www.gov. uk/government/uploads/system/uploads/attachment_data/file/213823/ dh_117794.pdf. Accessed July 2014.

9. Torrance $\mathrm{C}$, Wilson C. Consumer, user and carer involvement in health and social care [editorial]. Int J Consum Stud. 2010;34(5):493-494.

10. Beresford P. The theory and philosophy behind user involvement. In: Beresford P, Carr S, eds. Social Care, Service Users and User Involvement. London: Jessica Kingsley Publishers; 2012:21-36.

11. Croft S, Beresford P. The politics of participation. In: Taylor D, ed. Critical Social Policy: A Reader. London: Sage; 1996:175-198.

12. Boote J, Telford R, Cooper C. Consumer involvement in health research: a review and research agenda. Health Policy. 2002;61(2):213-236.

13. Macaulay AC, Commanda LE, Freeman WL, et al; North American Primary Care Research Group. Participatory research maximises community and lay involvement. BMJ. 1999;319(7212):774-778.

14. Boote J, Baird W, Sutton A. Public involvement in the systematic review process in health and social care: a narrative review of case examples. Health Policy. 2011;102(2-3):105-116.

15. International Collaboration for Participatory Health Research. Available from: http://www.icphr.org/. Accessed July 2014.

16. INVOLVE. Briefing note eight: Ways that people can be involved in the research cycle. Available from: http://www.invo.org.uk/posttyperesource/where-and-how-to-involve-in-the-research-cycle/. Accessed April 2013.

17. Tischler V, D'Silva K, Cheetham A, Goring M, Calton T. Involving patients in research: the challenge of patient-centredness. Int J Soc Psychiatry. 2010;56(6):623-633.

18. Barber R, Beresford P, Boote J, Cooper C, Faulkner A. Evaluating the impact of service user involvement on research: a prospective case study. Int J Consum Stud. 2011;35(6):609-615.

19. Smith E, Donovan S, Beresford P, et al. Getting ready for user involvement in a systematic review. Health Expect. 2009;12(2):197-208.

20. Wilson C, Fothergill A, Rees H. A potential model for the first all Wales mental health service user and carer-led research group. J Psychiatr Ment Health Nurs. 2010;17(1):31-38.

21. Morrow E, Ross F, Grocott P, Bennett J. A model and measure for quality service user involvement in health research. Int J Consum Stud. 2010;34(5):532-539.

22. INVOLVE. People and participation: How to put citizens at the heart of decision making. Available from: http://www.involve.org.uk/wpcontent/uploads/2011/03/People-and-Participation.pdf. Accessed July 2014.
23. Cotterell P, Harlow G, Morris C, et al. Service user involvement in cancer care: the impact on service users. Health Expect. 2011;14(2):159-169.

24. Brett J, Staniszewska S, Mockford C, et al. Mapping the impact of patient and public involvement on health and social care research: a systematic review. Health Expect. Epub 19 Jul 2012.

25. Porteous C, Rea D. Involving People/Cynnwys Pobl: an internal evaluation of infrastructure support for active involvement in health and social care research in Wales. Cardiff: Involving People/Cynnwys Pobl; 2009.

26. Cancer Research UK. Cancer Research UK's Research Strategy 2009/10 2013/14. London: Cancer Research UK; 2008. Available from: http:// www.cancerresearchuk.org/prod_consump/groups/cr_common/@ fre/@gen/documents/generalcontent/research-strategy-dnld-version. pdf. Accessed July 2014.

27. National Cancer Research Institute. Involving patients and the public in research. Available from: http://www.ncri.org.uk/default. asp? $\mathrm{s}=1 \& \mathrm{p}=11: 2013$. Accessed .

28. Nelson A, Byrne A, Griffiths G, et al. Valuing patient experience in the Wales Cancer Trials Unit: an action research project of credible patient involvement. Marie Curie Cancer Care Annual Research Conference; London; 2009.

29. Woodward A, Fitzgibbon J, Nelson A. Public and patient involvement: experiences of a Registered Research Group and a Trials Unit. Involving People Annual Meeting; February 2011; Swansea, UK.

30. Fitzgibbon J, Simon N, Nelson A. Research Partner Coordinator at the Wales Cancer Trials Unit: A cuckoo in the nest? INVOLVE 2012 Conference: Putting people first in research; November 2012; Nottingham, UK.

31. Vale C, Fitzgibbon J, Hanley B, et al. Public involvement in clinical trials: Supplement to the briefing notes for researchers. Eastleigh, United Kingdom; INVOLVE; 2012. Available from: http://www.invo.org.uk/ wp-content/uploads/2012/04/INVOLVEpublicinvolvementinclinicaltr ialsBriefingnotes2012.pdf. Accessed March 2012.

32. Neal R, Hurt C, Roberts K, et al. The ELCID Trial: A feasibility randomised control trial looking at the effect on lung cancer diagnosis of giving a Chest X-Ray to smokers aged over 60 with new chest symptoms. National Awareness and Early Diagnosis Initiative Research Conference; Cambridge Research Institute, UK; 2013.

33. Smith JD, Baillie J, Baglin T, et al. A feasibility study to inform the design of a randomized controlled trial to identify the most clinically and cost effective anticoagulation length with low molecular weight heparin in the treatment of cancer associated thrombosis (ALICAT): study protocol for a mixed-methods study. Trials. 2014;15(1):122.

34. Trial Forge. Trial Forge: a systematic approach to making trials more efficient. Available from: http://nebula.wsimg.com/dc736e 8283c135d db0e4f76bad683e62?AccessKeyId=FFE129220DD76BFA83F4\&disp osition=0\&alloworigin $=1$. Accessed July 2014 .

35. Barber R, Boote JD, Parry GD, Cooper CL, Yeeles P, Cook S. Can the impact of public involvement on research be evaluated? A mixed methods study. Health Expect. 2012;15(3):229-241.
Patient Preference and Adherence

\section{Publish your work in this journal}

Patient Preference and Adherence is an international, peer-reviewed, open access journal that focuses on the growing importance of patient preference and adherence throughout the therapeutic continuum. Patient satisfaction, acceptability, quality of life, compliance, persistence and their role in developing new therapeutic modalities and compounds to optimize

\section{Dovepress}

clinical outcomes for existing disease states are major areas of interest for the journal. This journal has been accepted for indexing on PubMed Central. The manuscript management system is completely online and includes a very quick and fair peer-review system, which is all easy to use. Visit http://www dovepress.com/testimonials.php to read real quotes from published authors. 